Research Article

\title{
Fault Diagnosis of Electric Impact Drills Based on Time-Varying Loudness and Logistic Regression
}

\author{
Yapeng Jing $\mathbb{D},{ }^{1}$ Haitao Su $\mathbb{D},{ }^{1,2}$ Shao Wang, ${ }^{1}$ Wenhua Gui, ${ }^{1}$ and Qing Guo $\mathbb{D}^{1,2}$ \\ ${ }^{1}$ School of Electronic Engineering and Automation, Guilin University of Electronic Technology, Guilin 541004, China \\ ${ }^{2}$ Guangxi Key Laboratory of Automatic Detecting and Instruments, Guilin University of Electronic Technology, \\ Guilin 541004, China \\ Correspondence should be addressed to Haitao Su; suhaitao@guet.edu.cn and Qing Guo; sxgq@guet.edu.cn
}

Received 26 December 2020; Revised 24 February 2021; Accepted 12 March 2021; Published 24 March 2021

Academic Editor: Liang Guo

Copyright (C) 2021 Yapeng Jing et al. This is an open access article distributed under the Creative Commons Attribution License, which permits unrestricted use, distribution, and reproduction in any medium, provided the original work is properly cited.

\begin{abstract}
As the main component of an electric impact drill, the gearbox is used to decelerate and transmit power; damage and failure to the gears often lead to the transmission system's failure. Therefore, as the core component of power transmission, the fault detection and diagnosis of gearbox devices have attracted increasing attention. This paper presents a psychoacoustic-based fault diagnosis method for gears of electric impact drills. The proposed methods employ acoustic signals and the time-varying loudness theory of psychoacoustic parameters. Two states of electric impact drills were analyzed: an electric impact drill with healthy gears and an electric impact drill with faulty gears. A feature extraction peak-to-average ratio (PAR) method based on the time-varying loudness spectrum was described and implemented to compute the feature vectors. The classification was carried out by applying logistic regression (LR). This paper provides the results of an acoustic analysis of electric impact drills. The results had a good recognition rate and the total accuracy of recognition of EIDs based on the PAR with LR was $97 \%$. This method simulates the human auditory perception to detect the gear components of an electric impact drill, which can replace the traditional artificial listening detection method.
\end{abstract}

\section{Introduction}

Electric impact drills (EIDs) are an electrical tool used for tightening and loosening bolts and nuts or drilling holes. They are mainly composed of a motor, gearbox, and keyless chuck. EIDs are a kind of rotating machinery that plays a crucial role in the machinery and construction industries. To prevent the failure of EIDs, manufacturers should strictly control their quality and they must be quality-tested before leaving the factory to ensure that there are no faults in the internal parts. The mechanical faults of EIDs have been previously studied [1]. This article focuses on gear failure in the two-stage gearbox. Specifically, we are concerned about the problem of broken gear teeth in this paper. We attributed all possible gear failures to one type of failure, so only a healthy EID and a failed EID were analyzed in this article. At present, the factory production line's commonly used detection method is to rely on professional workers to listen online. They then judge whether the product is faulty according to an abnormal sound during the EID's operation. The workers' listening based on their past experience is affected by many external factors, such as technical maturity, physical conditions, and personal subjectivity. As a result, the subjective feelings related to listening vary greatly. It is difficult to use a stable and consistent standard that judges whether there are faults in an EID. It is for this reason that it is hard to enhance the quality of products further. With the advancement of technology, efficient and accurate fault detection methods are urgently required by industrial production.

In the past few years, researchers in the industry have often used electric current signals, vibration signals, acoustic emission signals, and sound signals (produced by a microphone) to detect gear faults. The analysis of electric currents was developed in previous articles [2-6], and their results of current recognition were excellent. The current 
gear fault diagnosis method has limited applicability and is based on the motor stator current. Vibration signal analysis is an essential means of detecting gear failure [7-19]. Nonetheless, with regard to the structure of the measured object, the vibration signal may be hard to obtain. In addition, the shell of the machine under test will shield or change part of the mechanical vibration signal characteristics, especially for an EID. The current most prevalent gear fault diagnosis method is to analyze vibration combined with the acoustic emission (AE) signal [20-22], which improves the diagnosis capability; however, this detection method is very complicated and requires two types of suitable sensors, which increases the diagnosis cost.

In recent years, some scholars have developed gear fault diagnosis methods in conjunction with vibration and sound signals [23-26]. Others have directly used sound signals for diagnosis [27-29] and achieved good results. The abnormal sound produced by a mechanical structure, which can reflect some failure of the rotating mechanism, has always been a problem for manufacturers. Therefore, using the mechanical structure's abnormal sound to judge its failure is a feasible method. The audio signal generated by the vibration of a rotating machine has a robust corresponding relationship with the device's operating state. A slight change in the characteristic sound signal may indicate a change in the equipment's operating state. When the mechanical equipment operates normally, the sound signal appears as a combination of fundamental components and the resulting higher-order harmonics, and periodic attenuation waveforms appear in the time domain. When the equipment fails, the mechanical equipment failure point will become a new excitation source in the sound signal. These excitation sources will take part to turn around for a period of time, and the frequency and amplitude of the sound signal will change significantly. Therefore, we are confident that fault diagnosis technology based on acoustic signals will be popular. In traditional signal processing techniques, most of the relevant features are extracted from the time, frequency, and timefrequency domains of the signal. Nevertheless, there is no apparent difference between the analysis method and the vibration method, failing to reflect the advantages of acoustic signal analysis.

On the factory production test rig, the professional workers, based on their past experience, decide the presence of a fault by listening to the sound emitted by the electric drill. Based on similar principles of listening, here we use psychoacoustic technology to defect malfunctions of EIDs. We analyzed and evaluated the sounds produced by EIDs using psychoacoustic theory to detect faults. We describe the application of a psychoacoustic-based approach to EIDs. We analyzed 200 electric impact drills (100 healthy and 100 faulty). In total, two hundred acoustic signals were analyzed: healthy EIDs (Figures 1 and 2), EIDs with a faulty sun gear (Figure 3(a)), EIDs with a faulty ring gear (Figure 3(b)), and EIDs with a faulty planet gear (Figure $3(\mathrm{c})$ ). In Section 2, we introduce the proposed psychoacoustic approach of signal processing and describe the classification algorithm in detail. In Section 3, the recognition results are depicted. We discuss our findings in Section 4 and provide a conclusion in Section 5.

\section{Proposed Fault Diagnosis Method Based on Time-Varying Loudness}

The fault diagnosis method of EID was based on psychoacoustics. The proposed technique included four processes: acoustic signal capture, acoustic signal preprocessing, feature extraction, and classification. Specifically, LabVIEW software was used for acoustic signals catching, preprocessing, and extracting the data. First, we used an NI6343 acquisition card and CRY-333 measuring microphone set (a brand made in China, frequency response $3.15-20 \mathrm{kHz}$ and sensitivity $50 \mathrm{mV} / \mathrm{Pa}$ ) to record acoustic signals of the electric impact drill in use at a sampling rate of $51,200 \mathrm{~Hz}$, and the signal duration was $1.7 \mathrm{~s}$. The microphone was placed $20 \mathrm{~cm}$ from the supporting bracket of the EID and $8 \mathrm{~cm}$ from the upper edge of the bracket. Second, the recorded signals were preprocessed. The purpose of this step was to convert the voltage signal into the sound pressure signal, since NI 6343 cannot directly collect the audio signal. Therefore, the preprocessing method is to divide the received voltage signal by the sensitivity parameter of the microphone. Third, we used a time-varying loudness model to calculate the time-varying loudness and specific loudness of the preprocessed acoustic signals, and we proposed a peak-to-average ratio (PAR) fault diagnosis method on the basis of time-varying loudness. Last, we used the logistic regression (LR) classifier for pattern creation and classification. The developed fault diagnosis approach based on psychoacoustics is shown in Figure 4.

In the experimental design, we used a supporting bracket to fix the electric impact drill and a microprocessor to control operation of the electric impact drill. In addition, the microprocessor also integrated the power supply module of the microphone. The lithium battery supplied power to the microcontroller. The schematic diagram of the experimental setup to record sound signals is depicted in Figure 5(a) and the experimental setup of the electric impact drill is shown in Figure 5(b).

\subsection{The Time-Varying Loudness Theory of Psychoacoustic} Parameters. Psychoacoustic parameters are objective physical quantities that describe the degree of difference in the subjective human perception of sound, which can quantitatively reflect the difference in auditory perception and eliminate individual bias. Commonly used psychoacoustics parameters include loudness, sharpness, fluctuation, and roughness. Equations were developed to calculate a serial of parameters that describe the complex human perception of sound quality objectively [30]. Importantly, loudness is the basis for the calculation of other parameters. On this basis, we calculate the time-varying loudness in compliance with DIN 45631/A1:2010. The time-varying loudness is based on the steady-state loudness. The calculation step size is set as $2 \mathrm{~ms}$ in the standard so that the loudness calculation is carried out once every $2 \mathrm{~ms}$. The calculation of excitation mode and specific loudness within $2 \mathrm{~ms}$ is the same as that of steady loudness. The overall 


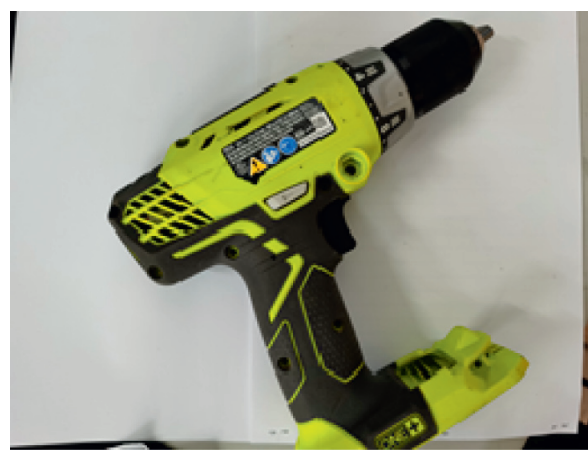

(a)

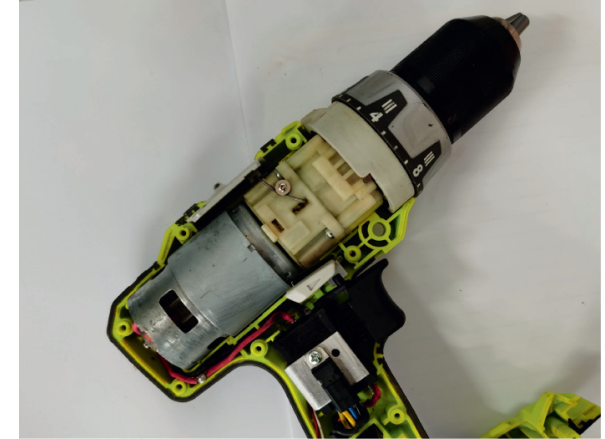

(b)

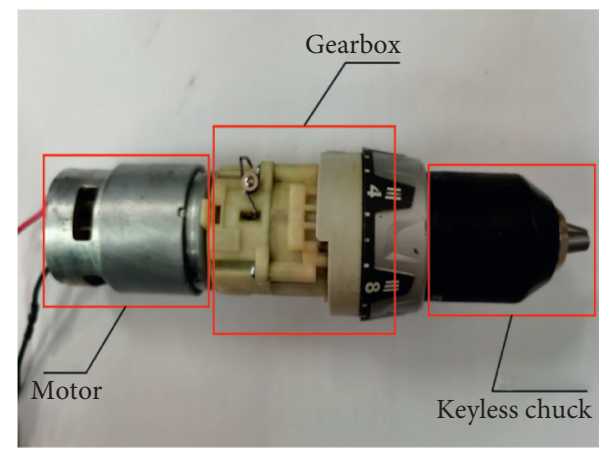

(c)

FIGURE 1: (a) Healthy electric impact drill (EID); (b) internal view of a healthy EID; (c) internal composition diagram of an EID, with the three main components (motor, gearbox, and keyless chuck) highlighted.

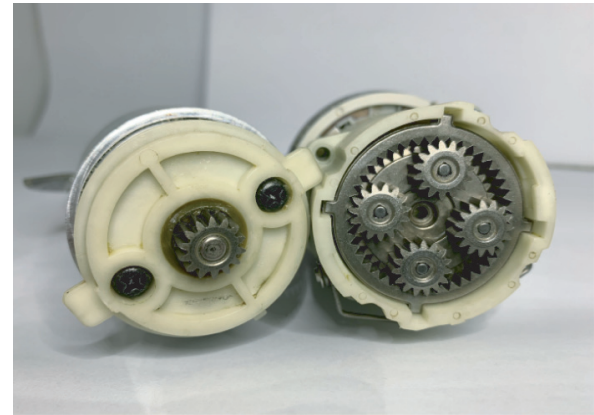

(a)

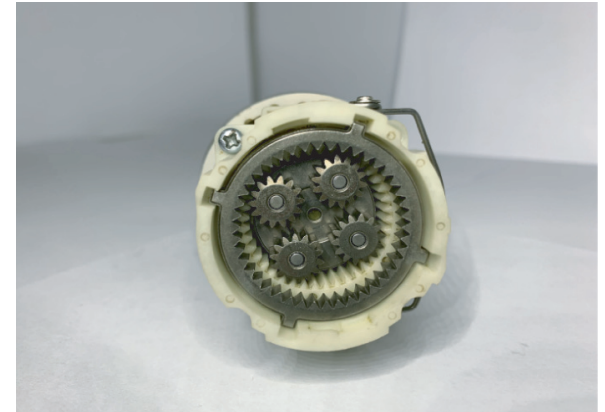

(b)

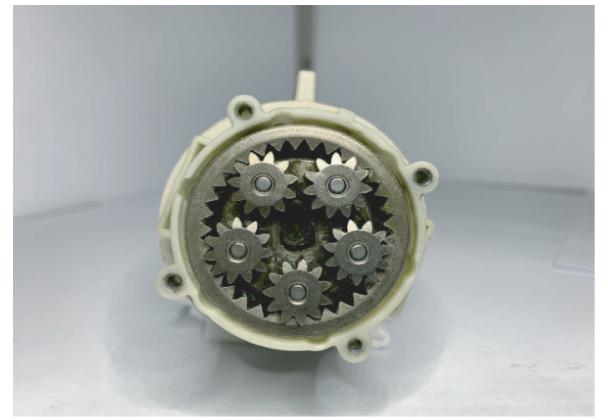

(c)

FIGURE 2: (a) Healthy EID with a working sun gear connecting the motor and gearbox. (b) Healthy EID with a working ring gear. (c) Healthy EID with a working planet gear. 


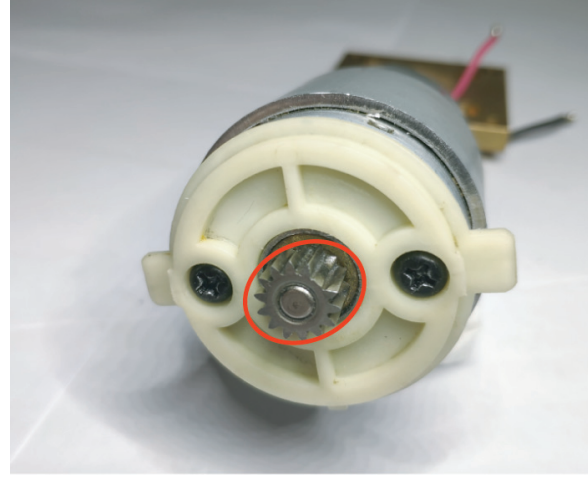

(a)

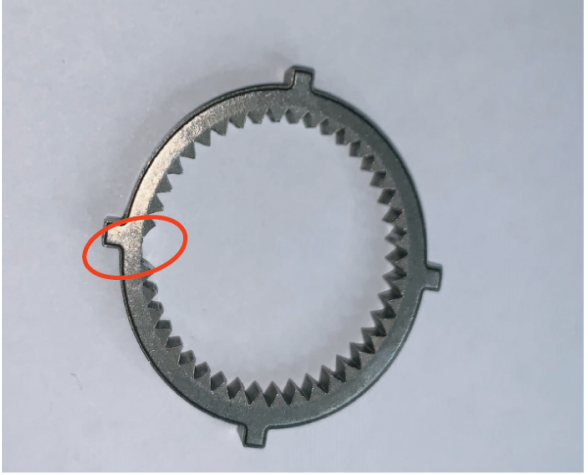

(b)

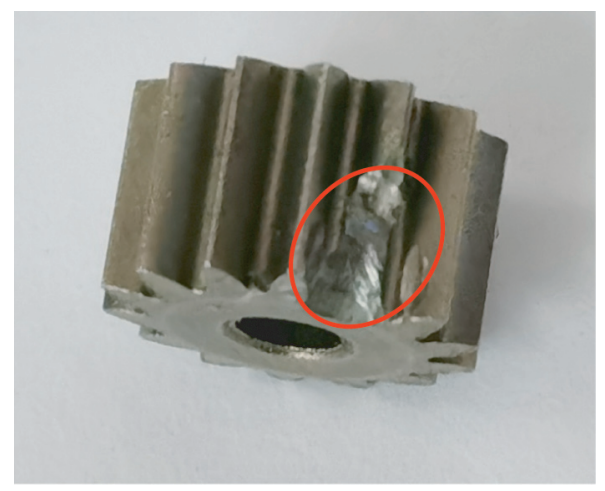

(c)

FIGURE 3: (a) EID with a faulty sun gear connecting the motor and gearbox; (b) EID with a faulty ring gear; (c) EID with a faulty planet gear. Faults are highlighted in the red ovals.

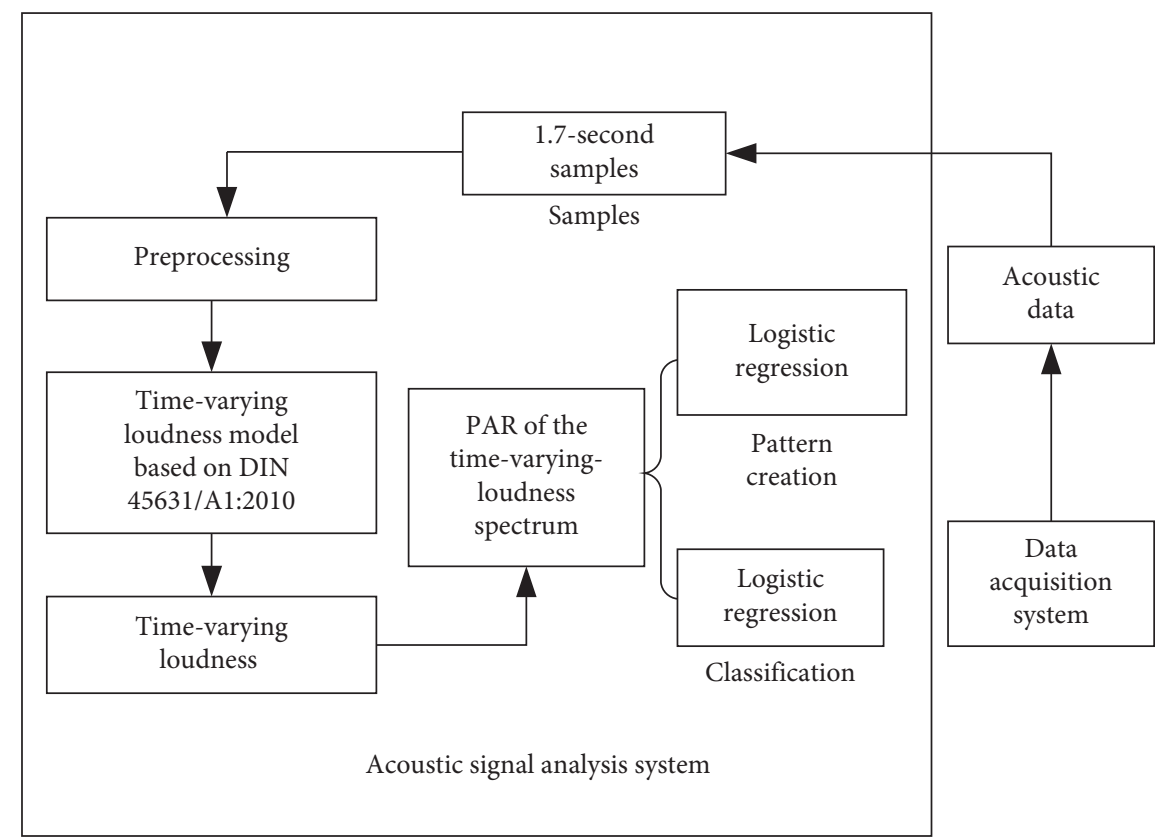

Figure 4: The proposed diagnostic system based on the acoustic signal.

loudness is obtained by summing the specific loudness in the whole frequency range. Here, we calculated and analyzed the time-varying specific loudness and found the difference between healthy products and faulty products. The loudness was obtained by integrating the time-varying specific loudness to get more accurate features of information. The 


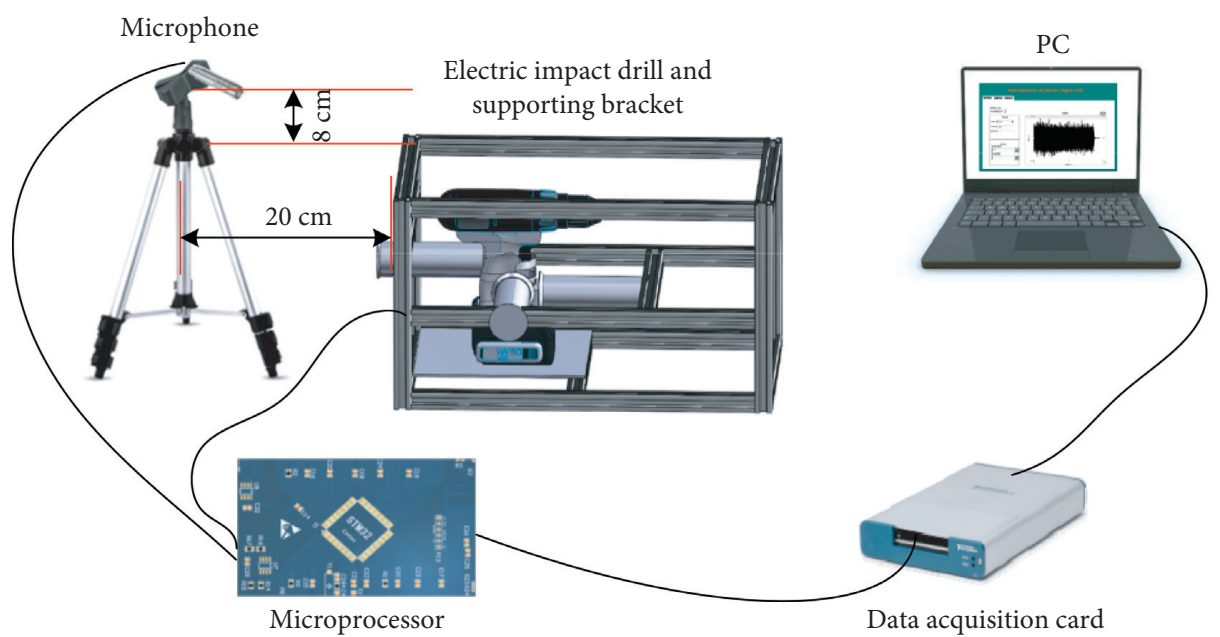

(a)

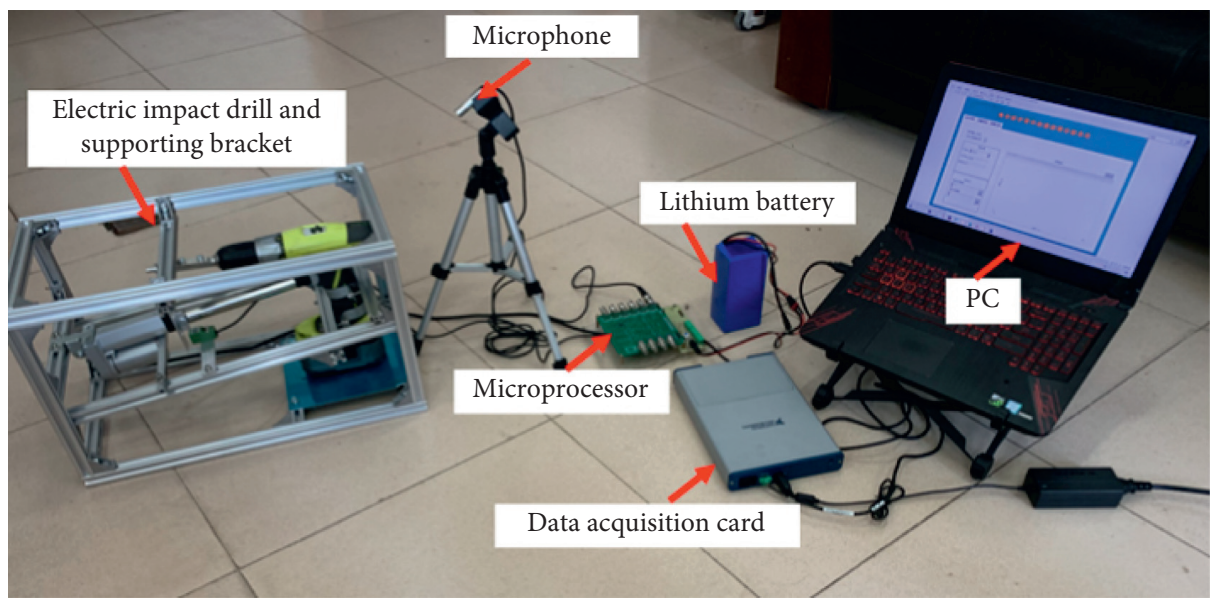

(b)

FIgURE 5: (a) Schematic diagram of the experimental setup and (b) experimental setup of the electric impact drill.

PAR calculation method based on the time-varying loudness spectrum is introduced below.

2.2. PAR of the Time-Varying Loudness Spectrum. The method of the PAR of the time-varying loudness spectrum was developed and implemented here. This feature extraction method was used differently in time-varying loudness. It consists of six signal processing steps:

(1) Calculate the time-varying loudness of the acoustic signals according to the time-varying loudness model in the German DIN 45631/A1:2010 standard.

(2) Intercept time-varying loudness data to obtain $y(t)$. We intercepted the loudness data from 0.3 seconds to 1.3 seconds for analysis to calculate the results more accurately, and we found that a $1 \mathrm{~s}$ sample was the best data size for analysis.

(3) Compute the FFT spectra of $y(t)$ to obtain $y(f)$, and compute the absolute values $|y(f)|$.

(4) Find the maximum value $A 1$ in the $[c, d]$ band and the corresponding frequency point $f_{1}$ of $A 1$. Then, calculate the lower critical frequency point $f_{2}$ and the upper critical frequency point $f_{3}$ according to the following formula:

$$
\begin{aligned}
& f_{2}= \begin{cases}c & f_{1}-\Delta f<c \\
f_{1}-\Delta f & f_{1}-\Delta f \geq c\end{cases} \\
& f_{3}= \begin{cases}f_{1}+\Delta f & f_{1}+\Delta f<d \\
d & f_{1}+\Delta f \geq d\end{cases}
\end{aligned}
$$

Finally, calculate the average value $A 2$ of $|y(f)|$ in frequency bands $\left[c, f_{2}\right]$ and $\left[f_{3}, d\right]$.

$$
A 2=\frac{\sum_{f=c}^{f_{2}}|y(f)|+\sum_{f=f_{3}}^{d}|y(f)|}{d-c-2 \Delta f},
$$

where $\Delta f=10, c=150, d=250 . \Delta f$ refers to the extended constant, which represents the statistical half-width of the peak where the maximum is located. In this step, $c$ and $d$ were selected based on the results of FFT analysis.

(5) According to $A 1$ and $A 2$ obtained in the fourth step, the PAR, $e$, was calculated by 


$$
e=\frac{|A 1|}{|A 2|} \times 100 \%
$$

(6) Form a feature vector $\left[e, f_{1}\right]$.

The approach used to build the PAR model based on the time-varying loudness spectrum is presented in Figure 6.

In this section, the analysis of the following three cases is described: Case 1 (Figure 7): healthy EID-a, faulty EID-a; Case 2 (Figure 8): healthy EID-b, faulty EID-b; Case 3 (Figure 9): healthy EID-c, faulty EID-c. Among them, faulty EID-a refers to the broken tooth of the sun gear connecting the motor and the gearbox, faulty EID-b refers to the broken tooth of the ring gear, and faulty EID-c refers to the broken tooth of the planet gear.

First, we calculated the time-varying specific loudness of different products and found apparent features. Timevarying loudness was calculated to distinguish more prominent features. Then, we analyzed the time-varying specific loudness, time-varying loudness, and time-varying loudness spectrum of healthy EIDs and faulty EIDs, respectively, and found that they have differences and regular changes. The most obvious difference of time-varying specific loudness is in subband $[14,24]$. The energy of healthy EID in subband $[14,24]$ is greater than that of faulty EID. The time-varying loudness is obtained by integrating the time-varying specific loudness on the time scale. We find that the time-varying loudness curve of healthy EID is stable and does not change greatly from $0.3 \mathrm{~s}$ to $1.3 \mathrm{~s}$, but there are many abrupt changes in the time-varying loudness curve of faulty EID. The most obvious difference between the spectra is mainly in the frequency band [150,250]. There are always one or more prominent peaks in the time-varying loudness spectrum of healthy EIDs in the $[150,250]$ frequency band; however, the faulty EID does not show a peak similar to the healthy EID. The acoustic signal, time-varying specific loudness, time-varying loudness, and time-varying loudness spectrum of the EID are depicted in Figures 7-9.

In the above three cases, the PAR of the healthy EID, $e$, was $14.68,30.16$, and 24.67 , respectively; the PAR of faulty EID, e, was 3.01, 3.08, and 2.86, respectively.

The classification was the last step in the identification of EID acoustic signals. Here, we used logistic regression as the classification tool for electric impact drill acoustic signal recognition. Logistic regression has been described in many scientific articles [31-33].

2.3. Logistic Regression. Logistic regression (LR) is one of the most commonly used and effective classification methods. It has been successfully applied in industry and has good interpretability and generalization. Besides, LR is used to model the probability of a certain category or event, which is based on probability to classify events. Like other supervised learning algorithms, the LR algorithm also uses the process of iterative optimization to find the best segmentation hyperplane after learning manually labeled training data. The LR model is a generalized linear model, which can be expressed as follows:

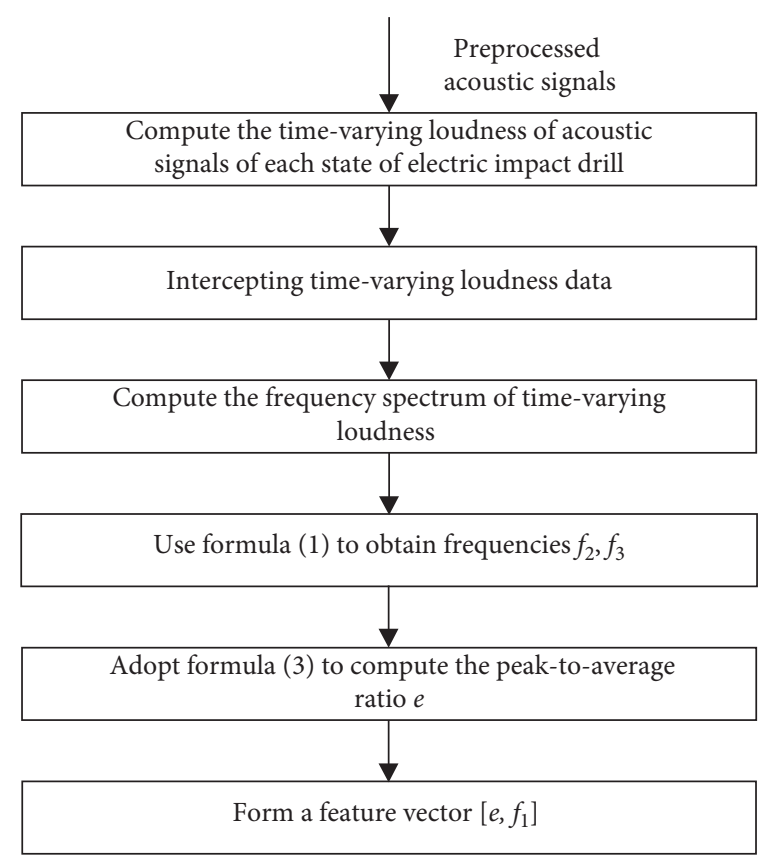

FIGURE 6: Flowchart of the peak-to-average ratio (PAR) model based on the time-varying loudness spectrum.

$$
\theta_{0}+\theta_{1} x_{1}+\theta_{2} x_{2}+\cdots+\theta_{n} x_{n}=\sum_{i=0}^{n} \theta_{i} x_{i}=\theta^{T} x
$$

where $\theta=\left(\theta_{0}, \theta_{1}, \theta_{2}, \ldots, \theta_{n}\right)$ is the feature weight vector and $x$ is the feature vector. In this paper, $\left[e, f_{1}\right]$ was used as the feature vector.

LR uses a probability function $P(y \mid x ; \theta)$ for prediction. In the probability function, $y$ is a discrete value and $x$ is a discrete or continuous quantity. In the binary classification problem, $y=\{0,1\}$. In this article, $y=0$ represents the faulty EID and $y=1$ represents the healthy EID. The LR model was expressed as follows:

$$
P(y \mid x ; \theta)=\frac{e^{y \theta^{T} x}}{1+e^{y \theta^{T} x}} .
$$

As the feature weight vector $\theta$ is not the optimal parameter, we employed the $\log$-likelihood function $J(\theta)(6)$ as the loss function of the LR model.

$$
J(\theta)=\frac{1}{n} \sum_{j=1}^{n}\left[-y_{j} \log \left(h_{\theta}\left(x_{j}\right)\right)-\left(1-y_{j}\right) \log \left(1-h_{\theta}\left(x_{j}\right)\right)\right] \text {, }
$$

where $h_{\theta}(x)=P(y \mid x ; \theta)$.

\section{Analysis of Acoustic Signals}

Acoustic signals were measured with two different states of the electric impact drill: A healthy electric impact drill and faulty electric impact drill with gear failure. The operating parameters were $18.0 \mathrm{~V}$ with a motor speed of $1600 \mathrm{r} / \mathrm{min}$. The acoustic signals of the electric impact drill in both states 


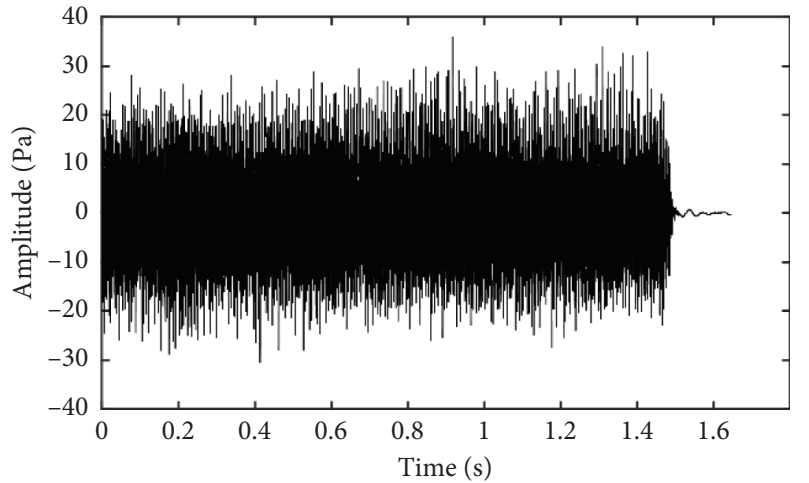

(a)

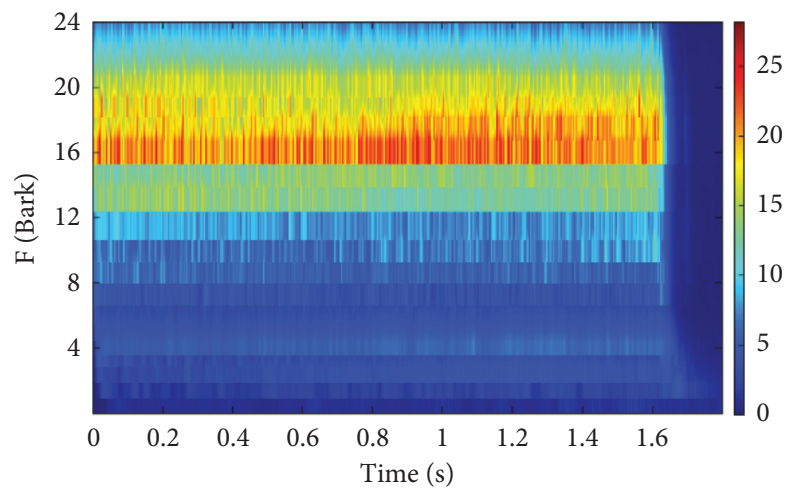

(c)

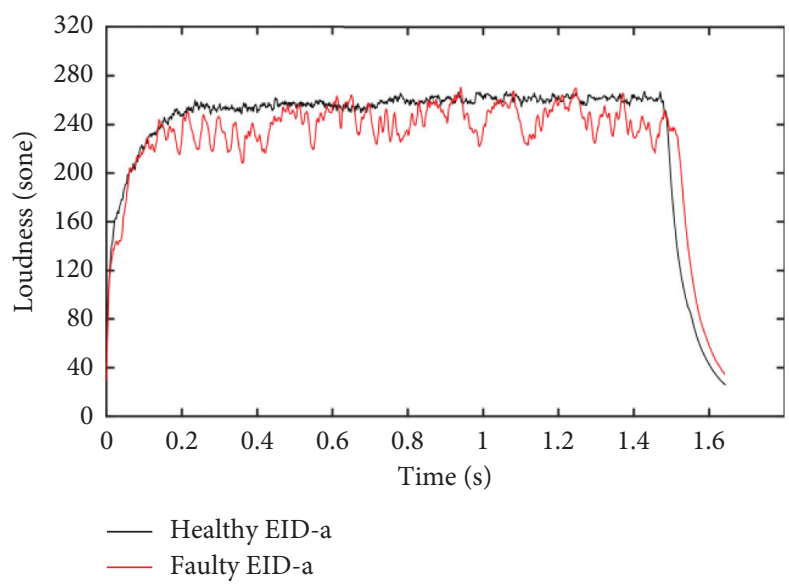

(e)

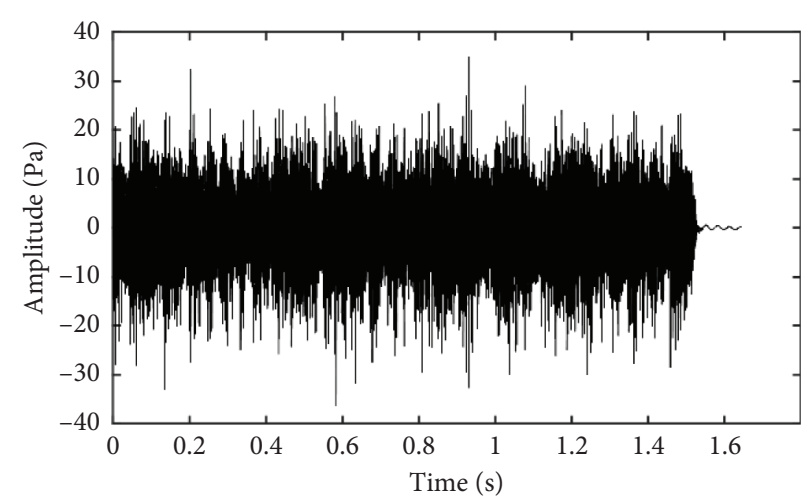

(b)

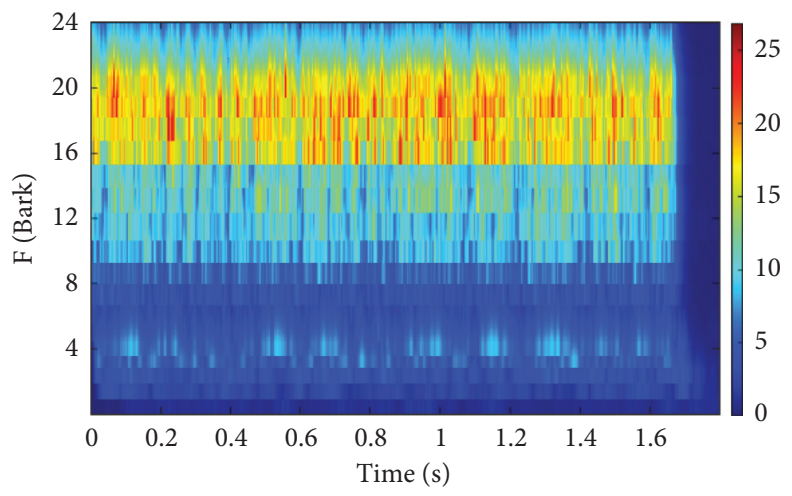

(d)

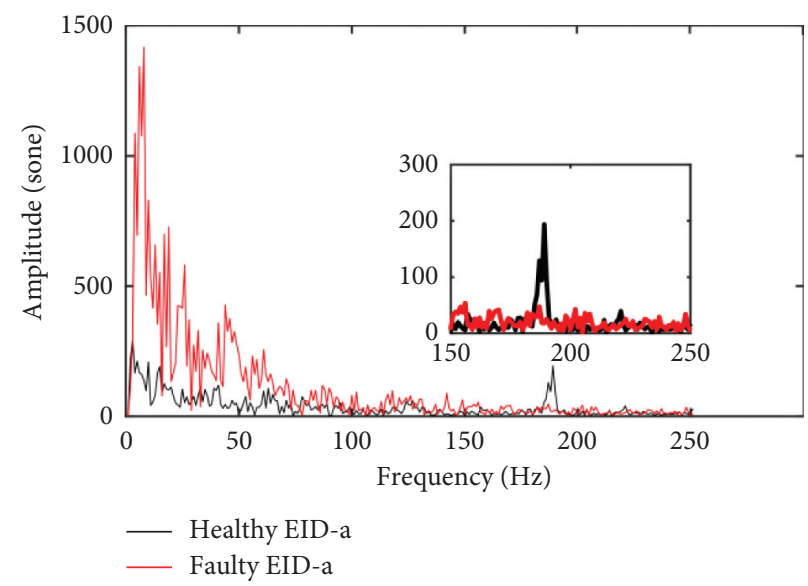

(f)

Figure 7: Acoustic signal analysis of Case 1: (a) acoustic signal of healthy EID-a, (b) acoustic signal of faulty EID-a, (c) time-varying specific loudness of healthy EID-a, (d) time-varying specific loudness of faulty EID-a, (e) time-varying loudness of healthy EID-a and faulty EID-a, and (f) time-varying loudness spectrum of healthy EID-a and faulty EID-a.

were analyzed using the proposed method, and the different acoustic signals were classified.

\subsection{Results Based on the PAR of the Time-Varying Loudness} Spectrum. We analyzed 10 faulty EIDs and healthy EIDs from 20 audio files in the training dataset, and the computed PAR values of time-varying loudness spectra of healthy EIDs and faulty EIDs are presented in Tables 1 and 2.
We can see that the PAR values of the healthy EIDs and the faulty EIDs are not similar in value. It will be easy to recognize these two classes.

3.2. Classification Results. We used 20 sound signal files for pattern creation and 200 sound signal files for the testing process. Training and test samples of audio signals of EIDs were analyzed using the proposed approach (Figure 6). The accuracy of LR recognition based on the PAR model was expressed as 


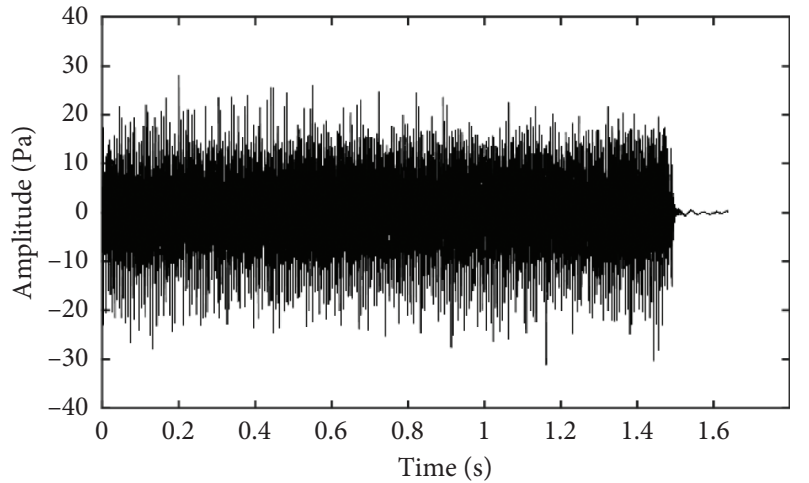

(a)

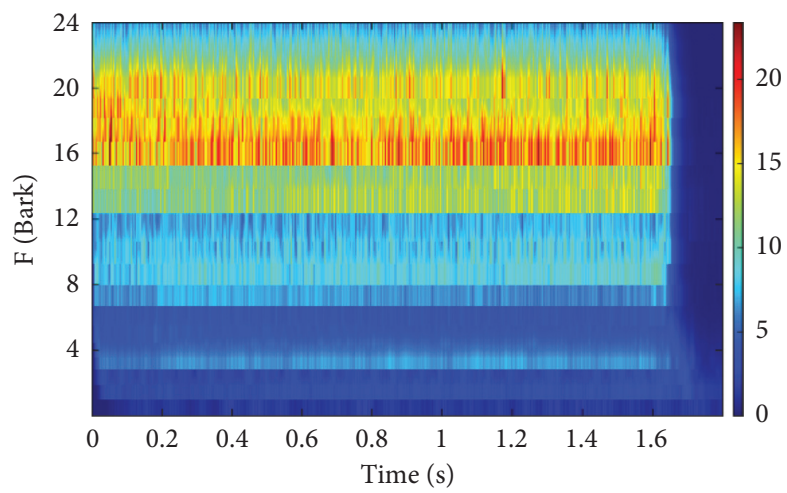

(c)

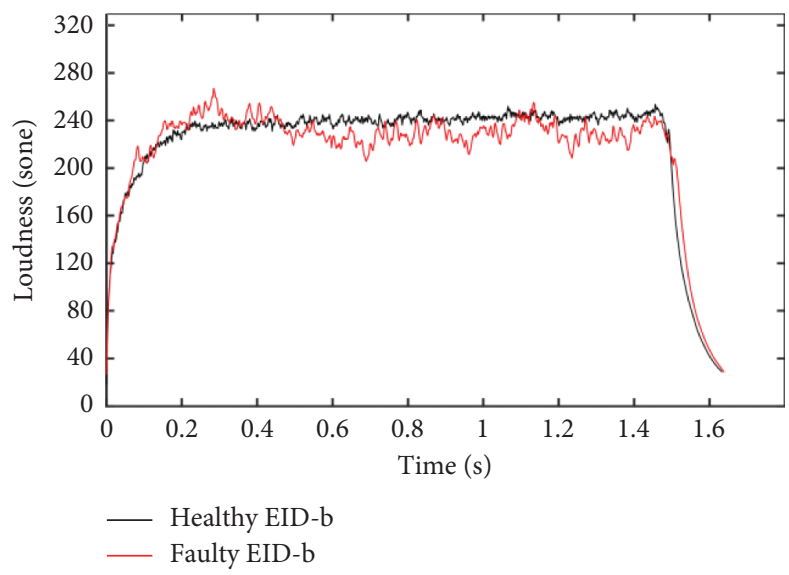

(e)

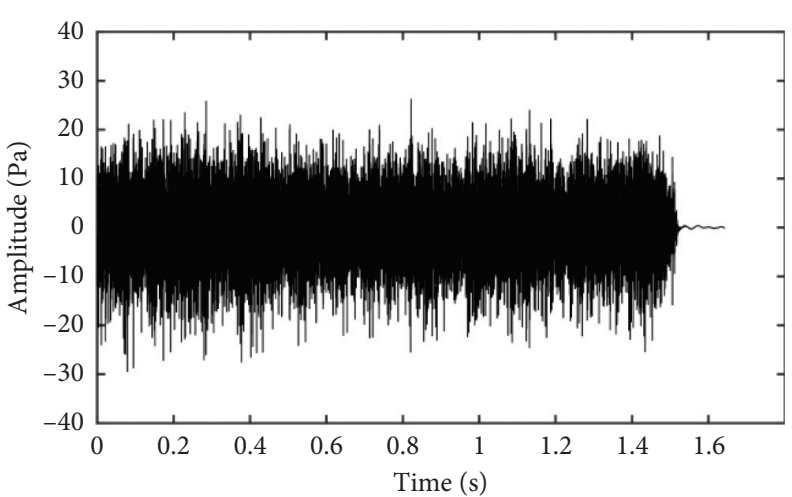

(b)

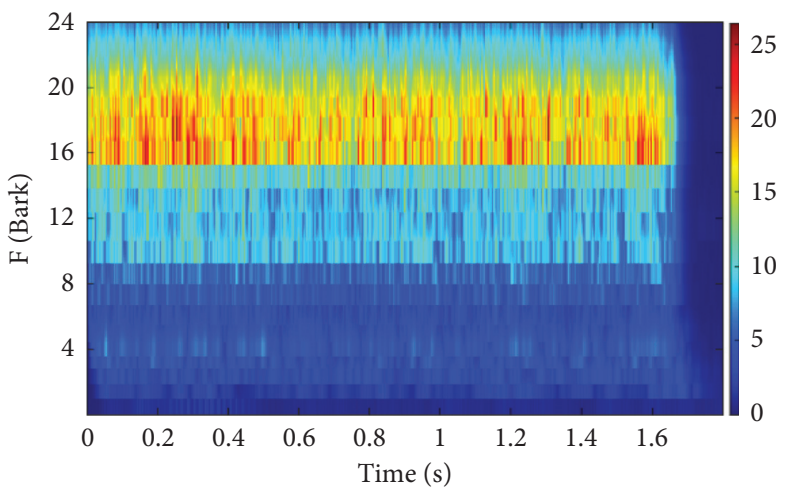

(d)

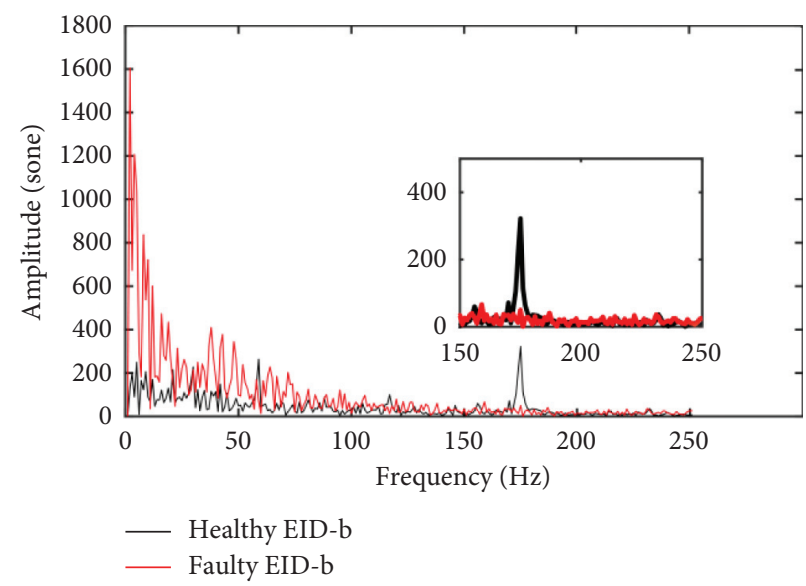

(f)

FIgURE 8: Acoustic signal analysis of Case 2: (a) acoustic signal of healthy EID-b, (b) acoustic signal of faulty EID-b, (c) time-varying specific loudness of healthy EID-b, (d) time-varying specific loudness of faulty EID-b, (e) time-varying loudness of healthy EID-b and faulty EID-b, and (f) time-varying loudness spectrum of healthy EID-b and faulty EID-b.

$$
\operatorname{Acc}_{\text {EID }}=\frac{\left(N_{\text {Pass }}\right)}{\left(N_{\text {Total test samples }}\right)},
$$

where $\mathrm{Acc}_{\mathrm{EID}}$ is the accuracy of recognition of acoustic signal of the selected class, $N_{\text {Pass }}$ is the number of test sound signal files class-tested properly, and $N_{\text {Total test samples }}$ is the number of all test sound signal files of the selected class.

The total accuracy of recognition was defined as follows:

$$
\mathrm{TACc}_{\mathrm{EID}}=\frac{\mathrm{Acc}_{\text {Healthy EIDs }}+\mathrm{Acc}_{\text {Faulty EIDs }}}{2},
$$

where $\mathrm{TACC}_{\mathrm{EID}}$ is the total accuracy of recognition of the EID, $\mathrm{Acc}_{\text {Healthy EIDs }}$ is the accuracy of recognition of healthy EIDs, and $\mathrm{Acc}_{\text {Faulty EIDs }}$ is the accuracy of recognition of faulty EIDs.

In this paper, we use 200 sound signal samples as test samples for classification, including 100 healthy EIDs and 


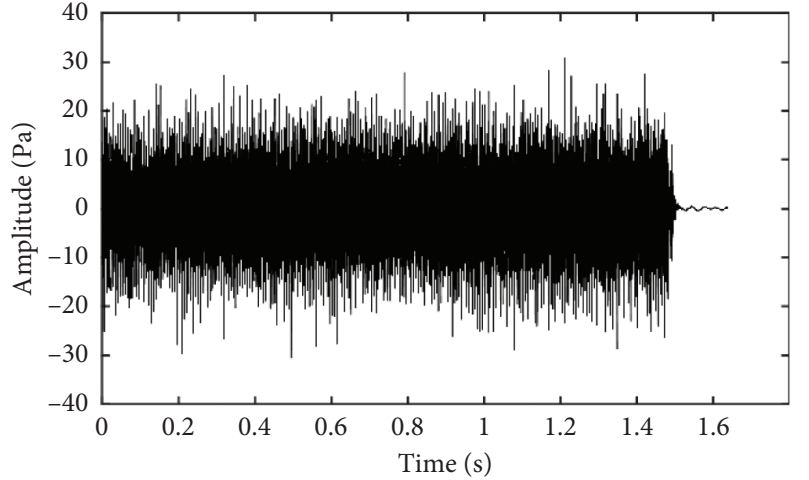

(a)

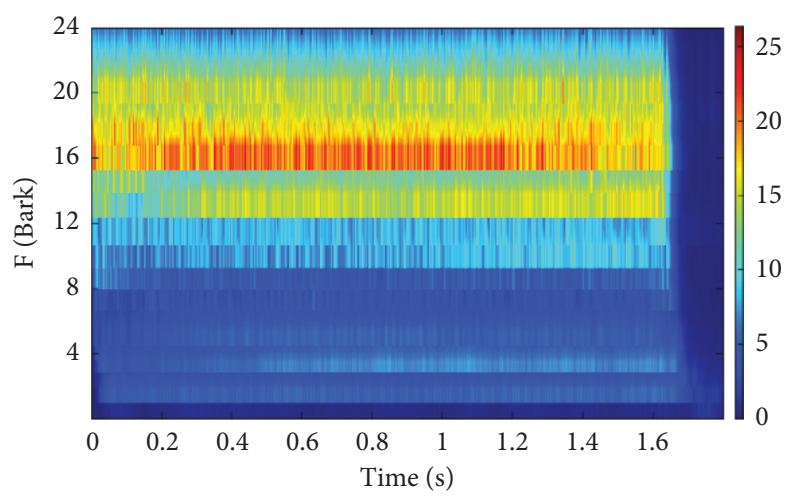

(c)

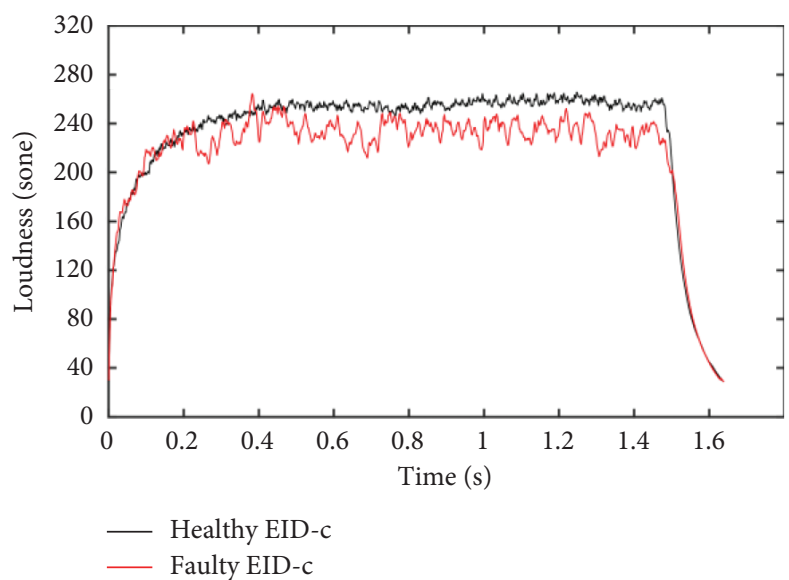

(e)

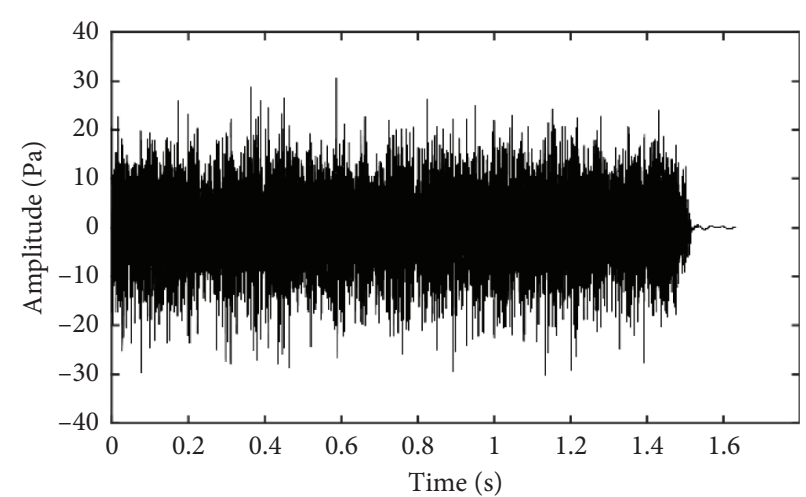

(b)

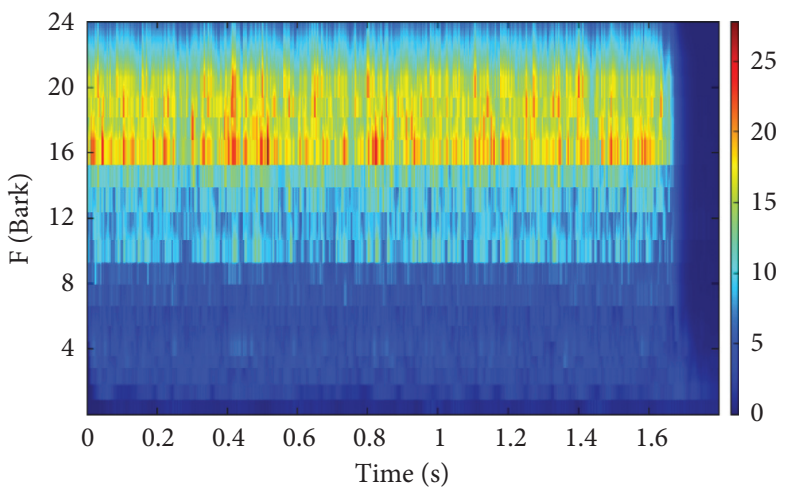

(d)

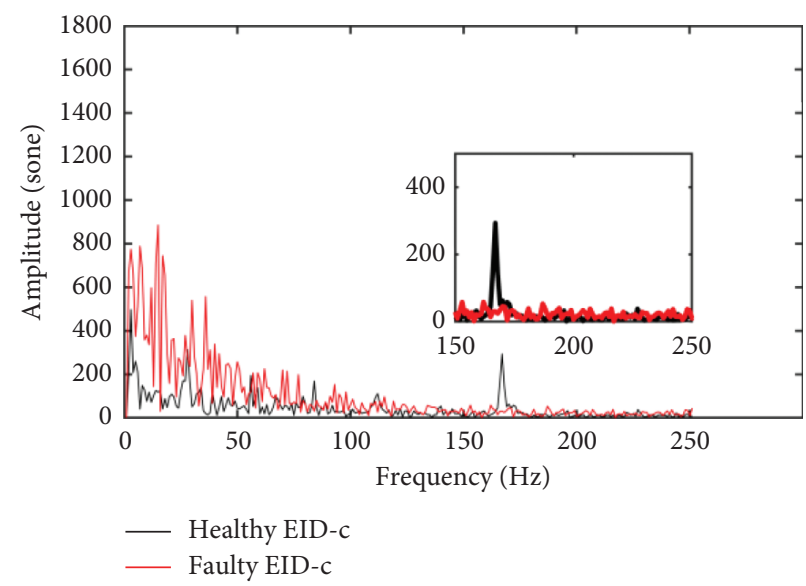

(f)

FIgURe 9: Acoustic signal analysis of Case 3: (a) acoustic signal of healthy EID-c, (b) acoustic signal of faulty EID-c, (c) time-varying specific loudness of healthy EID-c, (d) time-varying specific loudness of faulty EID-c, (e) time-varying loudness of healthy EID-c and faulty EID-c, and (f) time-varying loudness spectrum of healthy EID-c and faulty EID-c.

TABLE 1: PAR of the time-varying loudness spectra of healthy EIDs.

\begin{tabular}{lccccc}
\hline Samples & PAR value & $f_{1}(\mathrm{~Hz})$ & Samples & PAR value & $f_{1}(\mathrm{~Hz})$ \\
\hline 1 & 20.62 & 188 & 6 & 12.26 & 184 \\
2 & 14.68 & 188 & 7 & 9.91 & 194 \\
3 & 14.05 & 175 & 8 & 17.13 & 182 \\
4 & 10.75 & 181 & 9 & 9.53 & 184 \\
5 & 21.3 & 184 & 10 & 8.74 & 186 \\
\hline
\end{tabular}

TABLE 2: PAR of the time-varying loudness spectra of faulty EIDs.

\begin{tabular}{lccccc}
\hline Samples & PAR value & $f_{1}(\mathrm{~Hz})$ & Samples & PAR value & $f_{1}(\mathrm{~Hz})$ \\
\hline 1 & 3.1 & 171 & 6 & 3.75 & 160 \\
2 & 2.89 & 184 & 7 & 3.9 & 170 \\
3 & 2.37 & 184 & 8 & 2.98 & 162 \\
4 & 3.38 & 168 & 9 & 2.33 & 195 \\
5 & 2.7 & 160 & 10 & 3.01 & 168 \\
\hline
\end{tabular}


TABle 3: The classification accuracy on the training dataset and testing dataset using the PAR model with different classification methods.

\begin{tabular}{lcc}
\hline \multirow{2}{*}{ Methods } & \multicolumn{2}{c}{ Accuracy (\%) on } \\
& Training dataset & Testing dataset \\
\hline PAR + LR (our method) & 100 & 97 \\
PAR + SVM & 100 & 88 \\
PAR + KNN & 100 & 94.5 \\
\hline
\end{tabular}

100 faulty EIDs. We classify the EIDs using PAR method by different classifiers and calculate the classification results of the training dataset and testing dataset, as shown in Table 3. Finally, we also show the classification results of healthy EIDs and faulty EIDs using the peak-to-average ratio of time-varying loudness spectra and the LR classifier in the testing dataset; see Table 4.

From Table 3, we can see that the recognition results of the PAR model and LR classifier are the best. Moreover, the classification results of the Support Vector Machine (SVM) and K-Nearest Neighbor (KNN) classifier are not as good as LR because of parameter optimization and K-value selection.

Acoustic signals of the EIDs were processed by the PAR method and LR classifier (Table 4). The computed values of $\mathrm{Acc}_{\text {Healthy EIDs }}$ and $\mathrm{Acc}_{\text {Faulty EIDs }}$ were as follows: $\mathrm{Acc}_{\text {Healthy EIDs }}=94 \%$ and $\mathrm{Acc}_{\text {Faulty EIDs }}=100 \%$.

\section{Discussion}

The gear fault diagnosis scheme based on acoustic signals has limitations, as the collection of sound signals has rigorous requirements of the surrounding environment. The sound collection process for this study was conducted in a tranquil setting. Therefore, it is necessary to ensure that there is no substantial noise interference before experimenting when testing in a new environment.

Another point is that the fault diagnosis scheme we proposed was carried out at the end of the assembly line detection of the EID, which involved gear fault detection. We did not take other failures into account (e.g., motor failure and plastic casing failure). What is more, we only consider EID with a single fault. If we consider multiple faults of EID, we should obtain more dimensional signals. For example, we may combine vibration signal and sound signal for fault diagnosis.

The fault diagnosis method based on time-varying loudness and LR proposed in this article mainly involve time-varying specific loudness, time-varying loudness, the PAR of time-varying loudness spectrum, and LR classification algorithm. Among them, the most important step is the feature extraction process. We analyzed the PAR values in the $[150,250]$ frequency band of the time-varying loudness spectrum to distinguish between healthy and faulty products. If motor failures or other failures are considered, the $[150,250]$ subband may not meet the analysis conditions required. The appropriate frequency band should be selected for analysis based on the frequency difference of the time-varying loudness of functioning and defective products.
TABLE 4: The classification accuracy on the testing dataset using the PAR of the time-varying loudness spectrum and logistic regression (LR).

\begin{tabular}{lc}
\hline Type of acoustic signal & $\operatorname{Acc}_{\mathrm{EID}}(\%)$ \\
\hline Healthy EIDs & 94 \\
Faulty EIDs & 100 \\
The total accuracy of recognition of EIDs & TAcc $_{\mathrm{EID}}(\%) 97$ \\
\hline
\end{tabular}

\section{Conclusions}

In this paper, we presented a method for testing electric impact drills based on psychoacoustics. For this method, the acquisition of acoustic signals was carried out by a microphone and $\mathrm{NI}$ acquisition card; the primary processes of acoustic analysis included the preprocessing of acoustic signals and feature extraction and classification. The psychoacoustic parameters (time-varying loudness, time-varying specific loudness, and PAR) are the main features of the acoustic signal. The proposed method is the PAR time-varying loudness spectrum, and we applied an LR classifier for classification; its correct recognition rate was $97 \%$. Our analysis scheme, based on time-varying loudness, is simple and of low cost and can simultaneously detect a machine and perform the instant measurement and online monitoring of it. This advanced psychoacoustics-based method has many applications, such as for fault diagnosis in machine tool equipment. It can be used for motors, engines, machinery, and power tools. The proposed method based on psychoacoustics also has limitations, such as the presence of background noise and the reflection of sound. This fault diagnosis method based on psychoacoustics should be developed further in the future.

\section{Data Availability}

The data supporting the findings of this study can be obtained from the corresponding author as reasonably required.

\section{Conflicts of Interest}

The authors declare that there are no conflicts of interest.

\section{Acknowledgments}

This research was funded by the Innovation Project of GUET Graduate Education (Grant nos. 2020YCXS099 and 2020YCXS099), the Scientific Base and Special Talent Project of Guangxi of China under Grant Guike AD19110026, and Guangxi Natural Science Foundation of China (Grant no. 2018GXNSFAA294071).

\section{References}

[1] A. Glowacz, "fault detection of electric impact drills and coffee grinders using acoustic signals," Sensors (Basel), vol. 19, 2019.

[2] D. Lu, W. Qiao, and X. Gong, "Current-based gear fault detection for wind turbine gearboxes," IEEE Transactions on Sustainable Energy, vol. 8, no. 4, pp. 1453-1462, 2017. 
[3] X. Chen and Z. Feng, "Induction motor stator current analysis for planetary gearbox fault diagnosis under time-varying speed conditions," Mechanical Systems and Signal Processing, vol. 140, Article ID 106691, 2020.

[4] S. H. Kia, H. Henao, and G.-A. Capolino, "Gear tooth surface damage fault detection using induction machine stator current space vector analysis," IEEE Transactions on Industrial Electronics, vol. 62, no. 3, pp. 1866-1878, 2015.

[5] S. Rajagopalan, T. G. Habetler, R. G. Harley, T. Sebastian, and B. Lequesne, "Current/voltage-based detection of faults in gears coupled to electric motors," IEEE Transactions on Industry Applications, vol. 42, no. 6, pp. 1412-1420, 2006.

[6] N. Feki, G. Clerc, and P. Velex, "Gear and motor fault modeling and detection based on motor current analysis," Electric Power Systems Research, vol. 95, pp. 28-37, 2013.

[7] D. Xiao, J. Ding, X. Li, and L. Huang, "gear fault diagnosis based on kurtosis criterion VMD and SOM neural network," Applied Sciences, vol. 9, 2019.

[8] Z. Wu, Q. Zhang, L. Cheng, and S. Tan, "A new method of two-stage planetary gearbox fault detection based on multisensor information fusion," Applied Sciences, vol. 9, 2019.

[9] T. Wang, Q. Han, F. Chu, and Z. Feng, "Vibration based condition monitoring and fault diagnosis of wind turbine planetary gearbox: a review," Mechanical Systems and Signal Processing, vol. 126, pp. 662-685, 2019.

[10] L. Wang, "Fault diagnosis of a helical gearbox based on an adaptive empirical wavelet transform in combination with a spectral subtraction method," Applied Sciences, vol. 9, 2019.

[11] O. D. Mohammed, M. Rantatalo, J.-O. Aidanpää, and U. Kumar, "Vibration signal analysis for gear fault diagnosis with various crack progression scenarios," Mechanical Systems and Signal Processing, vol. 41, no. 1-2, pp. 176-195, 2013.

[12] Z. Feng, D. Zhang, and M. Zuo, "Planetary gearbox fault diagnosis via joint amplitude and frequency demodulation analysis based on variational mode decomposition," Applied Sciences, vol. 7, 2017.

[13] S. Xue and I. Howard, "Torsional vibration signal analysis as a diagnostic tool for planetary gear fault detection," Mechanical Systems and Signal Processing, vol. 100, pp. 706-728, 2018.

[14] K. Vernekar, H. Kumar, and K. V. Gangadharan, "Gear fault detection using vibration analysis and continuous wavelet transform," Procedia Materials Science, vol. 5, pp. 1846-1852, 2014.

[15] C. Li and M. Liang, "Time-frequency signal analysis for gearbox fault diagnosis using a generalized synchrosqueezing transform," Mechanical Systems and Signal Processing, vol. 26, pp. 205-217, 2012.

[16] V. Gunasegaran and V. Muralidharan, "Fault diagnosis of spur gear system through decision tree algorithm using vibration signal," Materials Today: Proceedings, vol. 22, pp. 3232-3239, 2020.

[17] Z. Feng and M. J. Zuo, "Vibration signal models for fault diagnosis of planetary gearboxes," Journal of Sound and Vibration, vol. 331, no. 22, pp. 4919-4939, 2012.

[18] L. S. Dhamande and M. B. Chaudhari, "Compound gearbearing fault feature extraction using statistical features based on time-frequency method," Measurement, vol. 125, pp. 6377, 2018.

[19] J. Cheng, Y. Yang, and D. Yu, "The envelope order spectrum based on generalized demodulation time-frequency analysis and its application to gear fault diagnosis," Mechanical Systems and Signal Processing, vol. 24, no. 2, pp. 508-521, 2010.

[20] C. Li, R.-V. Sanchez, G. Zurita, M. Cerrada, D. Cabrera, and R. E. Vásquez, "Gearbox fault diagnosis based on deep random forest fusion of acoustic and vibratory signals," Mechanical Systems and Signal Processing, vol. 76-77, pp. 283-293, 2016.

[21] S. Delvecchio, P. Bonfiglio, and F. Pompoli, "Vibro-acoustic condition monitoring of Internal Combustion Engines: a critical review of existing techniques," Mechanical Systems and Signal Processing, vol. 99, pp. 661-683, 2018.

[22] X. Li, J. Li, Y. Qu, and D. He, "Gear pitting fault diagnosis using integrated $\mathrm{CNN}$ and GRU network with both vibration and acoustic emission signals," Applied Sciences, vol. 9, no. 4, p. 768, 2019.

[23] D. P. Jena and S. N. Panigrahi, "Automatic gear and bearing fault localization using vibration and acoustic signals," $A p$ plied Acoustics, vol. 98, pp. 20-33, 2015.

[24] N. Baydar and A. Ball, "A comparative study of acoustic and vibration signals in detection of gear failures using wignerville distribution," Mechanical Systems and Signal Processing, vol. 15, no. 6, pp. 1091-1107, 2001.

[25] P. V. Kane and A. B. Andhare, "Critical evaluation and comparison of psychoacoustics, acoustics and vibration features for gear fault correlation and classification," Measurement, vol. 154, 2020.

[26] N. Baydar and A. Ball, "Detection of gear failures via vibration and acoustic signals using wavelet transform," Mechanical Systems and Signal Processing, vol. 17, no. 4, pp. 787-804, 2003.

[27] Y. Yao, H. Wang, S. Li et al., "End-to-end convolutional neural network model for gear fault diagnosis based on sound signals," Applied Sciences, vol. 8, 2018.

[28] A. Parey and A. Singh, "Gearbox fault diagnosis using acoustic signals, continuous wavelet transform and adaptive neurofuzzy inference system," Applied Acoustics, vol. 147, pp. 133-140, 2019.

[29] M. Amarnath and I. R. Praveen Krishna, "Local fault detection in helical gears via vibration and acoustic signals using EMD based statistical parameter analysis," Measurement, vol. 58, pp. 154-164, 2014.

[30] E. Zwicker and H. Fastl, Psychoacoustics: Facts and Models, Vol. 22, Springer Science \& Business Media, Berlin, Germany, 2013.

[31] J. Zhang, Y. Zhao, M. Liu, and L. Kong, "Bearings fault diagnosis based on adaptive local iterative filtering-multiscale permutation entropy and multinomial logistic model with group-lasso," Advances in Mechanical Engineering, vol. 11, 2019.

[32] H. Zhang, P. Chen, and Q. Wang, "Fault diagnosis method based on EEMD and multi-class logistic regression," in Proceedings of 2018 3rd International Conference on Smart City and Systems Engineering (ICSCSE), pp. 859-863, Xiamen, China, December 2018.

[33] D. H. Pandya, S. H. Upadhyay, and S. P. Harsha, "Fault diagnosis of rolling element bearing by using multinomial logistic regression and wavelet packet transform," Soft Computing, vol. 18, no. 2, pp. 255-266, 2013. 